\title{
Bayesian Analysis of the Survival Function and Failure Rate of Weibull Distribution with Censored Data
}

\author{
Chris Bambey Guure ${ }^{1}$ and Noor Akma Ibrahim ${ }^{1,2}$ \\ ${ }^{1}$ Institute for Mathematical Research, Universiti Putra Malaysia, 43400 Serdang, Selangor, Malaysia \\ ${ }^{2}$ Department of Mathematics, Universiti Putra Malaysia, 43400 Serdang, Selangor, Malaysia
}

Correspondence should be addressed to Chris Bambey Guure, bam.bey1@hotmail.com

Received 17 August 2012; Accepted 18 September 2012

Academic Editor: Carlo Cattani

Copyright (C) 2012 C. B. Guure and N. A. Ibrahim. This is an open access article distributed under the Creative Commons Attribution License, which permits unrestricted use, distribution, and reproduction in any medium, provided the original work is properly cited.

\begin{abstract}
The survival function of the Weibull distribution determines the probability that a unit or an individual will survive beyond a certain specified time while the failure rate is the rate at which a randomly selected individual known to be alive at time $(t-1)$ will die at time $(t)$. The classical approach for estimating the survival function and the failure rate is the maximum likelihood method. In this study, we strive to determine the best method, by comparing the classical maximum likelihood against the Bayesian estimators using an informative prior and a proposed data-dependent prior known as generalised noninformative prior. The Bayesian estimation is considered under three loss functions. Due to the complexity in dealing with the integrals using the Bayesian estimator, Lindley's approximation procedure is employed to reduce the ratio of the integrals. For the purpose of comparison, the mean squared error (MSE) and the absolute bias are obtained. This study is conducted via simulation by utilising different sample sizes. We observed from the study that the generalised prior we assumed performed better than the others under linear exponential loss function with respect to MSE and under general entropy loss function with respect to absolute bias.
\end{abstract}

\section{Introduction}

As a result of the adaptability in fitting time-to-failure of a very widespread multiplicity to multifaceted mechanisms, the Weibull distribution has assumed the centre stage especially in the field of life-testing and reliability/survival analysis. It has shown to be very useful for modelling and analysing life time data in medical, biological and engineering sciences, Lawless [1]. Much of the attractiveness of the Weibull distribution is due to the wide variety of shapes it can assume by altering its parameters.

Censoring is a feature that is recurrent in lifetime and reliability data analysis, it occurs when exact lifetimes or run-outs can only be collected for a portion of the inspection units. 
According to Rinne [2], "A data sample is said to be censored when, either by accident or design, the value of the variables under investigation is unobserved for some of the items in the sample."

Maximum likelihood estimator (MLE) is quiet efficient and very popular both in literature and practice. Bayesian approach has been employed for estimating parameters. Some research have been done to compare MLE and that of the Bayesian approach in estimating the survival function and the parameters of the Weibull distribution. Sinha [3] determined the Bayes estimates of the reliability function and the hazard rate of the Weibull failure time distribution by employing squared error loss function, AbdelWahid and Winterbottom [4] studied the approximate Bayesian estimates for the Weibull reliability function and hazard rate from censored data by employing a new method that has the potential of reducing the number of terms in Lindley procedure, and $\mathrm{Al}$ Omari and Ibrahim [5] conducted a study on Bayesian survival estimator for Weibull distribution with censored data using squared error loss function with Jeffreys prior amongst others.

Guure et al. [6] applied Bayesian estimation, for the two-parameter Weibull distribution using extension of Jeffreys' prior information with three loss functions, SyuanRong and Shuo-Jye [7] considered Bayesian estimation and prediction for Weibull model with progressive censoring. Other recent papers employing different models can be seen in Cattani and Ciancio [8], Wang et al. [9], Cattani [10,11]. Similar work can be seen in Gupta and Kundu [12] Zellner [13], Al-Aboud [14], Al-Athari [15], Cattani et al. [16] Pandey et al. [17], and a work on generalized exponential distribution: Bayesian estimations, Kundu and Gupta [18] which is somehow similar to the Weibull distribution.

The aim of this paper is twofold. First is to consider the maximum likelihood estimator of the survival function and the failure rate. In order to obtain the estimate of the survival function and the failure rate, we first need the MLE of the Weibull two parameters under consideration here. It is observed that the MLE cannot be obtained in analytical form, we therefore assumed the Newton-Raphson method to compute the MLE, and the method works quite well.

The second aim of this paper is to consider the Bayesian approach for the survival function and the failure rate. It is remarkable that most of the Bayesian inference procedures have been developed with the usual squared error loss function, which is symmetrical and associates equal importance to the losses due to overestimation and underestimation of equal magnitude according to Vasile et al. [19]. However, such applications may be restrictive in most situations of practical importance. For example, in the estimation of the failure rate, an overestimation is usually much more serious than an underestimation. In this case, the use of a symmetric loss function might be inappropriate as stated by Basu and Ebrahimi [20]. In this paper, we obtain the Bayes estimates under the LINEX loss function, general entropy loss function, and squared error loss function using Lindley's approximation technique via simulation study with informative prior and a generalised noninformative prior.

The rest of the paper is arranged as follows: Section 2 contains the derivative of the parameters based on which the survival function and the failure rate are determined under maximum likelihood estimator, Section 3 is the Bayesian estimation approach. This is followed by simulation study in Section 4. Results and discussion are in Sections 5 and 6 is the conclusion. 


\section{Maximum Likelihood Estimation}

Let $\left(t_{i}, \ldots, t_{n}\right)$ be the set of $n$ random lifetimes with respect to the Weibull distribution with $\alpha$ and $\beta$ as the parameters, where $\alpha$ is the scale parameter and $\beta$ the shape parameter.

The probability density function (pdf), the cumulative distribution function (cdf), and the survival function $S(\cdot)$ of Weibull are given, respectively, as

$$
\begin{gathered}
f(t ; \alpha, \beta)=\frac{\beta}{\alpha}\left(\frac{t}{\alpha}\right)^{\beta-1} \exp \left[-\left(\frac{t}{\alpha}\right)^{\beta}\right] \quad \forall t>0, \\
F(t ; \alpha, \beta)=1-\exp \left[-\left(\frac{t}{\alpha}\right)^{\beta}\right] \\
S(t ; \alpha, \beta)=\exp \left[-\left(\frac{t}{\alpha}\right)^{\beta}\right]
\end{gathered}
$$

The likelihood function is

$$
L\left(t_{i} ; \alpha, \beta, \delta\right)=\prod_{i=1}^{n}\left[f\left(t_{i}, \alpha, \beta\right)\right]^{\delta_{i}}\left[s\left(t_{i}, \alpha, \beta\right)\right]^{1-\delta_{i}},
$$

where $\delta_{i}=1$ is the failure time and $\delta_{i}=0$ is for censored observations. $S(\cdot)$ is the survival function.

The log-likelihood function of (2.2) is

$$
\ell=\sum_{i=1}^{n}\left[\delta_{i}\left[\ln (\beta)-\beta \ln (\alpha)+(\beta-1) \ln \left(t_{i}\right)\right]-\left(\frac{t_{i}}{\alpha}\right)^{\beta}\right]
$$

We differentiate (2.3) with respect to the unknown parameters and equal the resulting equation to zero as follows:

$$
\begin{gathered}
\frac{\partial \ell}{\partial \alpha}=-\frac{\beta \sum_{i=1}^{n} \delta_{i}}{\alpha}+\frac{\beta \sum_{i=1}^{n}\left(t_{i} / \alpha\right)^{\beta}}{\alpha}=0 \\
\frac{\partial \ell}{\partial \beta}=\frac{\sum_{i=1}^{n} \delta_{i}}{\beta}-\sum_{i=1}^{n} \delta_{i} \ln (\alpha)+\sum_{i=1}^{n} \delta_{i} \ln \left(t_{i}\right)-\sum_{i=1}^{n}\left(\frac{t_{i}}{\alpha}\right)^{\beta} \ln \left(\frac{t_{i}}{\alpha}\right)=0
\end{gathered}
$$

The maximum likelihood estimator of $\alpha$ is

$$
\widehat{\alpha}_{M L}=\left(\frac{\sum_{i=1}^{n}\left(t_{i}\right)^{\widehat{\beta}}}{\sum_{i=1}^{n} \delta_{i}}\right)^{1 / \widehat{\beta}}
$$

The shape parameter $\widehat{\beta}$ is obtained by the method of Newton-Raphson since it cannot be solved analytically. 
The estimates of the survival function and the failure rate of the Weibull distribution under MLE are

$$
\begin{aligned}
& \widehat{S}_{\mathrm{ML}}(t)=\exp \left[-\left(\frac{t}{\widehat{\alpha}_{\mathrm{ML}}}\right)^{\widehat{\beta}_{\mathrm{ML}}}\right] \\
& \widehat{F}_{\mathrm{ML}}(t)=\frac{\widehat{\beta}_{\mathrm{ML}}}{\widehat{\alpha}_{\mathrm{ML}}}\left(\frac{t}{\widehat{\alpha}_{\mathrm{ML}}}\right)^{\hat{\beta}_{\mathrm{ML}}-1} .
\end{aligned}
$$

\section{Bayesian Inference}

In the field of statistics, Bayesian inference is viewed as a method that employs the Bayes' rule in other to update the probability estimate of a hypothesis taking into consideration new evidence as they become available. Bayesian updating is one of the essential techniques used in modern statistics, more importantly in mathematical statistics. Bayesian updating is particularly important in analysing sequence of data. Bayesian inference is applicable in many fields today, for example, engineering, medicine, accounting, and others. Bayes makes use of our prior beliefs of the parameters which is known as the prior distribution. It is the distribution of the parameters before any data is observed and is given as $p(\theta)$. It also takes into consideration the observed data which is viewed as the likelihood function and given as $L(t \mid \theta)$.

Bayesian inference is based on the posterior distribution which is simply the ratio of the joint density function to the marginal distribution.

The posterior distribution is

$$
\pi^{*}(\theta \mid t) \propto \frac{p(\theta) L(t \mid \theta)}{\int_{0}^{\infty} p(\theta) L(t \mid \theta) d \theta}
$$

where $t$ is the data and $\theta$ is the parameter of interest.

The Bayes estimator is considered under three loss functions which is also indisputable in Bayesian estimation. They are asymmetric (LINEX and general entropy) loss functions and symmetric (squared error) loss function.

Prior distribution of the parameters need to be assumed for the Bayesian inference. As discussed by Berger and Sun [21] and subsequently by Banerjee and Kundu [22], we let $\alpha$ take on a Gamma $(a, b)$ prior with $a>0$ and $b>0$. We assume that the prior of $\beta$ is independent of the prior of $\alpha$ and is in the neighbourhood of $(0, \infty)$. Let $v(\beta)$ represent the prior of $\beta$ and $v_{1}(\alpha)$ for $\alpha$, where

$$
\text { Gamma }(\alpha, \beta)=\frac{\beta^{\alpha}}{\Gamma(\alpha)} t^{\alpha-1} \exp (-\beta t)
$$

Let the likelihood equation which is $L\left(t_{i} \mid \alpha, \beta\right)$ be the same as (2.2).

The joint posterior density function of $(\alpha, \beta)$ is given by

$$
\pi(\alpha, \beta \mid t) \alpha L(t \mid \alpha, \beta) v(\beta) v_{1}(\alpha)
$$


The posterior probability density function of $\alpha$ and $\beta$ given the data $\left(t_{1}, t_{2}, \ldots, t_{n}\right)$ is obtained by dividing the joint posterior density function over the marginal distribution function as

$$
\pi^{*}(\alpha, \beta \mid t)=\frac{L(t \mid \alpha, \beta) v(\beta) v_{1}(\alpha)}{\iint_{0}^{\infty} L(t \mid \alpha, \beta) v(\beta) v_{1}(\alpha) d \alpha d \beta}
$$

\subsection{Generalised Noninformative Prior}

Our proposition of the generalised noninformative prior for the parameters $\alpha$ and $\beta$ is given such that

$$
v_{2}(\alpha, \beta)=\left(\frac{1}{\widehat{\alpha} \widehat{\beta}}\right)^{a}, \quad a>0
$$

We are assuming there is no or little knowledge on the parameters being estimated, where $\widehat{\alpha}$ and $\widehat{\beta}$ are the estimates from maximum likelihood with respect to the available data obtained by the researcher and $a$ is a constant that can assume any value in order to minimise the prior effect on the posterior distribution. We refer to this approach as the data-dependent prior and it is more or less an empirical or objective Bayes prior. This is an interesting new developing theory of objective priors and, while data dependent, it does not involve an inappropriate double use of the data, Berger [23], unless the sample size is fairly small. The generalised noninformative prior must not be misconstrued to imply a joint prior for the two parameters Weibull distribution. In other words, the scale and shape parameters are independent a priori.

The likelihood function from (2.2) is

$$
L\left(\mathrm{t}_{\mathrm{i}} \mid \alpha, \beta\right)=\prod_{i=1}^{n}\left\{\left(\frac{\beta}{\alpha}\right)\left(\frac{t_{i}}{\alpha}\right)^{\beta-1} \exp \left[-\left(\frac{t_{i}}{\alpha}\right)^{\beta}\right]\right\}^{\delta_{i}}\left\{\exp \left[-\left(\frac{t_{i}}{\alpha}\right)^{\beta}\right]\right\}^{1-\delta_{i}}
$$

With Bayes theorem the joint posterior distribution of the parameters $\alpha$ and $\beta$ is

$$
\begin{aligned}
& \pi(\alpha, \beta \mid t) \alpha L(t \mid \alpha, \beta) v_{2}(\alpha, \beta) \\
& \quad=k v_{2}(\alpha, \beta) \prod_{i=1}^{n}\left\{\left(\frac{\beta}{\alpha}\right)\left(\frac{t_{i}}{\alpha}\right)^{\beta-1} \exp \left[-\left(\frac{t_{i}}{\alpha}\right)^{\beta}\right]\right\}^{\delta_{i}}\left\{\exp \left[-\left(\frac{t_{i}}{\alpha}\right)^{\beta}\right]\right\}^{1-\delta_{i}},
\end{aligned}
$$

where $k$ is the normalizing constant that makes $\pi$ a proper pdf.

The posterior density function is obtained by using (3.4).

\subsection{Linear Exponential Loss Function}

This loss function according to Soliman et al. [24] rises approximately exponentially on one side of zero and approximately linearly on the other side. 
The posterior expectation of the LINEX loss function according to Zellner [13] is

$$
E_{\theta}[L(\widehat{\theta}-\theta)] \alpha \exp (c \hat{\theta}) E_{\theta}[\exp (-c \theta)]-c\left[\hat{\theta}-E_{\theta}(\theta)\right]-1
$$

with $E_{\theta}(\cdot)$ denoting the posterior expectation with respect to the posterior density of $\theta$. Therefore, the Bayes estimator of $\theta$, which is denoted by $\widehat{\theta}_{\mathrm{BL}}$ under LINEX loss function is the value of $\hat{\theta}$ which minimizes (3.8) and is

$$
\widehat{\theta}_{\mathrm{BL}}=-\frac{1}{c} \ln \left\{E_{\theta}[\exp (-c \theta)]\right\}
$$

provided that $E_{\theta}[\exp (-c \theta)]$ exist and is finite.

The posterior density of the survival function and the failure rate under this loss function are given as

$$
\begin{aligned}
S(t)_{\mathrm{BL}} & =E\left\{\exp \left[-c \exp \left[-\left(\frac{t_{i}}{\alpha}\right)^{\beta}\right]\right] \mid t\right\} \\
& =\frac{\iint_{0}^{\infty} v(\beta) v_{1}(\alpha) \exp \left[-c \exp \left[-\left(t_{i} / \alpha\right)^{\beta}\right]\right] L\left(t_{i} \mid \alpha, \beta\right) d \alpha d \beta}{\iint_{0}^{\infty} v(\beta) v_{1}(\alpha) L\left(t_{i} \mid \alpha, \beta\right) d \alpha d \beta}, \\
F(t)_{\mathrm{BL}} & =E\left\{\left[-c \exp \left(\frac{\beta}{\alpha}\right)\left(\frac{t}{\alpha}\right)^{\beta-1}\right] \mid t\right\} \\
& =\frac{\iint_{0}^{\infty} v(\beta) v_{1}(\alpha) \exp \left[-c(\beta / \alpha)(t / \alpha)^{\beta-1}\right] L\left(t_{i} \mid \alpha, \beta\right) d \alpha d \beta}{\iint_{0}^{\infty} v(\beta) v_{1}(\alpha) L\left(t_{i} \mid \alpha, \beta\right) d \alpha d \beta} .
\end{aligned}
$$

It can be observed that (3.10) contain ratio of integrals which cannot be obtained analytically and as a result we make use of Lindley approximation procedure to evaluate the integrals involved.

\subsection{Lindley Approximation}

A prior of $\beta$ need to be specified here so as to calculate the approximate Bayes estimates of $\alpha$ and $\beta$. Having specified a prior for $\alpha$ as Gamma $(a, b)$, it is similarly assumed that $v(\beta)$ also takes on a Gamma $(c, d)$ prior.

Lindley [25] proposed a ratio of integral of the form

$$
\frac{\int \omega(\theta) \exp \{L(\theta)\} d \theta}{\int v(\theta) \exp \{L(\theta)\} d \theta}
$$


where $L(\theta)$ is the log-likelihood and $\omega(\theta), v(\theta)$ are arbitrary functions of $\theta$. Assuming that $v(\theta)$ is the prior distribution for $\theta$ and $\omega(\theta)=u(\theta) \cdot v(\theta)$ with $u(\theta)$ being some function of interest.

The posterior expectation according to Sinha [3] is

$$
E\{u(\theta) \mid t\}=\frac{\int v(\theta) \exp \{L(\theta)+\rho(\theta)\} d(\theta)}{\int \exp \{L(\theta)+\rho(\theta)\} d(\theta)}
$$

where $\rho(\theta)=\log \{v(\theta)\}$.

This can be approximated asymptotically by

$$
E\{u(\theta) \mid x\}=\left[u+\frac{1}{2} \sum_{i} \sum_{j}\left(u_{i j}+2 u_{i} \cdot \rho_{j}\right) \cdot \sigma_{i j}+\frac{1}{2} \sum_{i} \sum_{j} \sum_{k} \sum_{l} L_{i j k} \cdot \sigma_{i j} \cdot \sigma_{k l} \cdot u_{l}\right],
$$

where $i, j, k, l=1,2, \ldots, n ; \theta=\left(\theta_{1}, \theta_{2}, \ldots, \theta_{m}\right)$.

Taking the two parameters into consideration, (3.13) reduces to

$$
\widehat{\theta}=u+\frac{1}{2}\left[\left(u_{11} \sigma_{11}\right)+\left(u_{22} \sigma_{22}\right)\right]+u_{1} \rho_{1} \sigma_{11}+u_{2} \rho_{2} \sigma_{22}+\frac{1}{2}\left[\left(L_{30} u_{1} \sigma_{11}^{2}\right)+\left(L_{03} u_{2} \sigma_{22}^{2}\right)\right],
$$

where $L$ is the log-likelihood equation in (2.3).

Taking the survival function, where

$$
\begin{aligned}
& u= \exp \left\{-c \exp \left[-\left(\frac{t}{\alpha}\right)^{\beta}\right]\right\}, \quad q=\exp \left[-\left(\frac{t}{\alpha}\right)^{\beta}\right], \\
& u_{1}=\frac{\partial u}{\partial \alpha}=c\left(\frac{\beta}{\alpha}\right)\left(\frac{-t}{\alpha}\right)^{\beta} q u, \\
& u_{11}=\frac{\partial^{2} u}{\partial \alpha^{2}}=-c\left(\frac{\beta^{2}}{\alpha^{2}}\right)\left[-\left(\frac{t}{\alpha}\right)^{\beta}\right] q u-c\left(\frac{\beta}{\alpha^{2}}\right)\left[-\left(\frac{t}{\alpha}\right)^{\beta}\right] q u \\
& \quad-c\left(\frac{\beta^{2}}{\alpha^{2}}\right)\left[-\left(\frac{t}{\alpha}\right)^{\beta}\right]^{2} q u+c^{2}\left(\frac{\beta^{2}}{\alpha^{2}}\right)\left[-\left(\frac{t}{\alpha}\right)^{\beta}\right]^{2} q^{2} u .
\end{aligned}
$$

In a similar approach $u_{2}=\partial u / \partial \beta$ and $u_{22}=\partial^{2} u / \partial \beta^{2}$ can be obtained. 
For the failure rate,

$$
\begin{gathered}
u=\exp \left[-c\left(\frac{\beta}{\alpha}\right)\left(\frac{t}{\alpha}\right)^{\beta-1}\right], \quad p=\left(\frac{\beta}{\alpha}\right)\left(\frac{t}{\alpha}\right)^{\beta-1}, \\
u_{2}=\frac{\partial u}{\partial \beta}=\left[-c p \ln \left(\frac{t}{\alpha}\right)-\left(\frac{c}{\alpha}\right)\left(\frac{t}{\alpha}\right)^{\beta-1}\right] u, \\
u_{22}=\frac{\partial^{2} u}{\partial \beta^{2}}=\left[-c p \ln ^{2}\left(\frac{t}{\alpha}\right)-2\left(\frac{c}{\alpha}\right)\left(\frac{t}{\alpha}\right)^{\beta-1} \ln \left(\frac{t}{\alpha}\right)\right] u \\
+\left[-c p \ln \left(\frac{t}{\alpha}\right)-\left(\frac{c}{\alpha}\right)\left(\frac{t}{\alpha}\right)^{\beta-1}\right]^{2} u
\end{gathered}
$$

$u_{1}=\partial u / \partial \alpha$ and $u_{11}=\partial^{2} u / \partial \alpha^{2}$ follow in like manner.

Let

$$
\begin{gathered}
\rho=\ln v_{1}(\alpha)+\ln v(\beta), \\
\rho_{1}=\frac{c-1}{\alpha}-d, \quad \rho_{2}=\frac{a-1}{\beta}-b, \\
L_{20}=\frac{\partial^{2} L}{\partial \alpha^{2}}=\frac{\beta \sum_{i=1}^{n} \delta_{i}}{\alpha^{2}}-\frac{\beta^{2} \sum_{i=1}^{n}\left(t_{i} / \alpha\right)^{\beta}}{\alpha^{2}}-\frac{\beta \sum_{i=1}^{n}\left(t_{i} / \alpha\right)^{\beta}}{\alpha^{2}}, \\
\sigma_{11}=\left(-L_{20}\right)^{-1}, \\
L_{30}=-\frac{2 \beta \sum_{i=1}^{n} \delta_{i}}{\alpha^{3}}+\frac{\beta^{3} \sum_{i=1}^{n}\left(t_{i} / \alpha\right)^{\beta}}{\alpha^{3}}+\frac{3 \beta^{2} \sum_{i=1}^{n}\left(t_{i} / \alpha\right)^{\beta}}{\alpha^{3}}+\frac{2 \beta \sum_{i=1}^{n}\left(t_{i} / \alpha\right)^{\beta}}{\alpha^{3}}, \\
L_{02}=-\frac{\sum_{i=1}^{n} \delta_{i}}{\beta^{2}}-\sum_{i=1}^{n}\left(\frac{t_{i}}{\alpha}\right)^{\beta} \ln \left(\frac{t_{i}}{\alpha}\right)^{2}, \\
\sigma_{22}=\left(-L_{02}\right)^{-1}, \\
L_{03}=\frac{\partial^{3} L}{\partial \beta^{3}}=\frac{2 \sum_{i=1}^{n} \delta_{i}}{\beta^{3}}-\sum_{i=1}^{n}\left(\frac{t_{i}}{\alpha}\right)^{\beta} \ln \left(\frac{t_{i}}{\alpha}\right)^{3} .
\end{gathered}
$$

\subsection{General Entropy Loss Function}

This is used to determine the degree of overestimation and underestimation of the parameters. It is simply a generalization of the entropy loss function.

The Bayes estimator of $\theta$, denoted by $\widehat{\theta}_{\mathrm{BG}}$ is given as

$$
\widehat{\theta}_{\mathrm{BG}}=\left[E_{\theta}(\theta)^{-k}\right]^{-1 / k}
$$

provided $E_{\theta}\left(\theta^{-k}\right)$ exist and is finite. 
The posterior density function of the survival function and the failure rate under general entropy loss are given, respectively, as

$$
\begin{aligned}
S(t)_{\mathrm{BG}} & =E\left\{\left[\exp \left[-\left(\frac{t}{\alpha}\right)^{\beta}\right]\right]^{-k} \mid t\right\} \\
& =\frac{\iint_{0}^{\infty} v(\beta) v_{1}(\alpha)\left[\exp \left[-(t / \alpha)^{\beta}\right]\right]^{-k} L\left(t_{i} \mid \alpha, \beta\right) d \alpha d \beta}{\iint_{0}^{\infty} v(\beta) v_{1}(\alpha) L\left(t_{i} \mid \alpha, \beta\right) d \alpha d \beta}, \\
F(t)_{\mathrm{BG}} & =E\left\{\left[\left(\frac{\beta}{\alpha}\right)\left(\frac{t}{\alpha}\right)^{\beta-1}\right]^{-k} \mid t\right\} \\
& =\frac{\iint_{0}^{\infty} v(\beta) v_{1}(\alpha)\left[(\beta / \alpha)(t / \alpha)^{\beta-1}\right]^{-k} L\left(t_{i} \mid \alpha, \beta\right) d \alpha d \beta}{\iint_{0}^{\infty} v(\beta) v_{1}(\alpha) L\left(t_{i} \mid \alpha, \beta\right) d \alpha d \beta} .
\end{aligned}
$$

By making use of Lindley procedure as in (3.14), where $u_{1}, u_{11}$, and $u_{2}, u_{22}$ represent the first and second derivatives of the survival function and the failure rate, the following equations are obtained:

$$
\begin{aligned}
u & =\left\{\exp \left[-\left(\frac{t}{\alpha}\right)^{\beta}\right]\right\}^{-k}, \quad e=\left[-\left(\frac{t}{\alpha}\right)^{\beta}\right] \\
u_{1} & =\frac{\partial u}{\partial \alpha}=u k\left(\frac{\beta}{\alpha}\right) e, \\
u_{11} & =\frac{\partial^{2} u}{\partial \alpha^{2}}=u k^{2}\left(\frac{\beta^{2}}{\alpha^{2}}\right) e^{2}-k\left(\frac{\beta^{2}}{\alpha^{2}}\right) e u-k\left(\frac{\beta}{\alpha^{2}}\right) e u .
\end{aligned}
$$

Hence, $u_{2}=\partial u / \partial \beta$ and $u_{22}=\partial^{2} u / \partial \beta^{2}$ follows.

For the failure rate,

$$
\begin{aligned}
u & =\left[\left(\frac{\beta}{\alpha}\right)\left(\frac{t}{\alpha}\right)^{\beta-1}\right]^{-k}, \quad r=\left(\frac{t}{\alpha}\right)^{\beta-1}, \\
u_{1} & =\frac{\partial u}{\partial \alpha}=-\frac{u k\left[-\left(\beta / \alpha^{2}\right) r-(\beta-1)\left(\beta / \alpha^{2}\right) r\right] \alpha}{\beta r}
\end{aligned}
$$




$$
\begin{aligned}
u_{11}=\frac{\partial^{2} u}{\partial \alpha^{2}}= & \frac{u k^{2}\left[-\left(\beta / \alpha^{2}\right) r-(\beta-1)\left(\beta / \alpha^{2}\right) r\right]^{2} \alpha^{2}}{\beta^{2} r^{2}} \\
& -\frac{u k\left[2\left(\beta / \alpha^{3}\right) r+3\left(\beta / \alpha^{3}\right)(\beta-1) r+\left(\beta / \alpha^{3}\right) r(\beta-1)^{2}\right] \alpha}{\beta r} \\
& -\frac{u k\left[-\left(\beta / \alpha^{2}\right) r-(\beta-1)\left(\beta / \alpha^{2}\right) r\right]}{\beta r} \\
& -\frac{u k\left[-\left(\beta / \alpha^{2}\right) r-(\beta-1)\left(\beta / \alpha^{2}\right) r\right](\beta-1)}{\beta r}
\end{aligned}
$$

With the same approach as given above $u_{22}=\partial u / \partial \beta$ and $u_{22}=\partial^{2} u / \partial \beta^{2}$ are obtained.

\subsection{Squared Error Loss Function}

The most common loss function used for Bayesian estimation is the squared error (SE), also called quadratic loss. The square error loss denotes the punishment in using $\widehat{\theta}$ to estimate $\theta$ and is given as $E(t \mid \theta)=[\widehat{\theta}(t)-\theta]^{2}$, where the expectation is taken over the joint distribution of $\theta$ and $(t)$. The posterior density function of the survival function and the failure rate under the Symmetric loss function are given as

$$
\begin{aligned}
S(t)_{\mathrm{BS}} & =E\left\{\exp \left[-\left(\frac{t}{\alpha}\right)^{\beta}\right] \mid t\right\} \\
& =\frac{\iint_{0}^{\infty} v(\beta) v_{1}(\alpha)\left[\exp \left[-(t / \alpha)^{\beta}\right]\right] L\left(t_{i} \mid \alpha, \beta\right) d \alpha d \beta}{\iint_{0}^{\infty} v(\beta) v_{1}(\alpha) L\left(t_{i} \mid \alpha, \beta\right) d \alpha d \beta}, \\
F(t)_{\mathrm{BS}} & =E\left[\left(\frac{\beta}{\alpha}\right)\left(\frac{t}{\alpha}\right)^{\beta-1} \mid t\right] \\
& =\frac{\iint_{0}^{\infty} v(\beta) v_{1}(\alpha)\left[(\beta / \alpha)(t / \alpha)^{\beta-1}\right] L\left(t_{i} \mid \alpha, \beta\right) d \alpha d \beta}{\iint_{0}^{\infty} v(\beta) v_{1}(\alpha) L\left(t_{i} \mid \alpha, \beta\right) d \alpha d \beta} .
\end{aligned}
$$
Function

Applying the same Lindley approach here as in (3.14), we have for the Survival

$$
\begin{aligned}
& u=\exp \left[-\left(\frac{t}{\alpha}\right)^{\beta}\right], \quad u_{1}=\frac{\partial u}{\partial \alpha}=-\left(\frac{\beta}{\alpha}\right) e u \\
& u_{11}=\frac{\partial^{2} u}{\partial \alpha^{2}}=\left(\frac{\beta}{\alpha^{2}}\right) e u+\left(\frac{\beta^{2}}{\alpha^{2}}\right) e u+\left(\frac{\beta^{2}}{\alpha^{2}}\right) e^{2} u .
\end{aligned}
$$


In a similar approach $u_{2}=\partial u / \partial \beta$ and $u_{22}=\partial^{2} u / \partial \beta^{2}$ can be obtained.

For the Failure Rate,

$$
\begin{gathered}
u=\left(\frac{\beta}{\alpha}\right)\left(\frac{t}{\alpha}\right)^{\beta-1}, \quad d=\ln \left(\frac{t}{\alpha}\right), \\
u_{2}=\frac{\partial u}{\partial \beta}=\left(\frac{1}{\alpha}\right) r+u d, \quad u_{22}=\frac{\partial^{2} u}{\partial \beta^{2}}=\left(\frac{2}{\alpha}\right) r d^{2}+u d .
\end{gathered}
$$

$u_{1}=\partial u / \partial \alpha$ and $u_{11}=\partial^{2} u / \partial \alpha^{2}$ follow in like manner.

With respect to the generalised noninformative prior, the same procedures as above are also employed but $\rho=\ln v_{1}(\alpha)+\ln v(\beta)$ is substituted by $\rho=\ln \left[v_{2}(\alpha, \beta)\right]$.

\section{Simulation Study}

We have considered in this simulation study a sample size of $n=25,50$ and 100, which is representative of small, moderate, and large data sets. The following steps were employed to generate the data.

A lifetime $T$ is generated from the sample sizes indicated above from the Weibull distribution which represents failure of the product or unit. The values of the assumed actual parameters of the Weibull distribution were taken to be $\alpha=0.5$ and 1.5 and that of $\beta=0.8$ and 1.2. The same sample size is generated from the uniform distribution for the censored time $C$ with $(0, b)$, where the value of $b$ depends solely on the proportion of the observations that are censored. In our study, we consider the percentage of censoring to be $30 . T=\min (T, C)$ is taken as the minimum of the failure time and the censored time of the observed time $T$, where

$$
T= \begin{cases}\delta_{i}=1, & \text { if } X \leq C, \\ \delta_{i}=0, & \text { if } X>C .\end{cases}
$$

To compute the Bayes estimates, an assumption is made such that $\alpha$ and $\beta$ take, respectively, Gamma $(a, b)$ and Gamma $(c, d)$ priors. We set the hyperparameters to zero, that is, $a=b=c=d=0$ in order to obtain noninformative priors. Note that at this point, the priors become nonproper but the results do not have any significant difference with the implementation of proper priors as stated by Banerjee and Kundu [22].

The values for the loss parameters of both the LINEX and general entropy were $c=$ $k= \pm 1$ and \pm 2 . For problems on how to choose the loss parameter values, see Calabria and Pulcini [26]. We have also considered the generalised noninformative prior to be $a=3$ and 5 without loss of generality. These were iterated $(R) 1000$ times. The mean squared error and the absolute bias values are determined and presented below for the purpose of comparison. Consider the following:

$$
\operatorname{MSE}(\hat{\theta})=\frac{\sum_{r=1}^{R}\left(\hat{\theta}^{r}-\theta\right)^{2}}{R-1}, \quad \operatorname{Abs} \cdot \operatorname{Bias}(\hat{\theta})=\frac{\sum_{r=1}^{R}\left|\hat{\theta}^{r}-\theta\right|}{R-1}
$$


Table 1: MSEs and Abs. Biases (in parenthesis) for the survival function $S(t)$.

\begin{tabular}{|c|c|c|c|c|c|}
\hline \multirow{2}{*}{$n$} & & \multicolumn{2}{|c|}{$\alpha=0.5$} & \multicolumn{2}{|c|}{$\alpha=1.5$} \\
\hline & & $\beta=0.8$ & $\beta=1.2$ & $\beta=0.8$ & $\beta=1.2$ \\
\hline \multirow{10}{*}{25} & ML & $0.01577(0.09892)$ & $0.01613(0.10131)$ & $0.01644(0.10076)$ & $0.01635(0.10273)$ \\
\hline & BS & $0.01581(0.09909)$ & $0.01618(0.10149)$ & $0.01648(0.10092)$ & $0.01639(0.10290)$ \\
\hline & $\mathrm{BL}(c=1)$ & $0.01681(0.10213)$ & $0.01731(0.10556)$ & $0.01564(0.09971)$ & 0.01707 (0.10299) \\
\hline & $\mathrm{BL}(c=-1)$ & $0.01613(0.10035)$ & $0.01732(0.10361)$ & $0.01625(0.10014)$ & $0.01687(0.10428)$ \\
\hline & $\mathrm{BL}(c=2)$ & $0.01604(0.09968)$ & $0.01742(0.10428)$ & $0.01629(0.10079)$ & $0.01711(0.10437)$ \\
\hline & $\mathrm{BL}(c=-2)$ & $0.01623(0.10091)$ & $0.01753(0.10573)$ & $0.01578(0.09839)$ & $0.01708(0.10266)$ \\
\hline & $\mathrm{BG}(k=1)$ & $0.01568(0.09809)$ & $0.01770(0.10566)$ & $0.01520(0.09737)$ & $0.01667(0.10269)$ \\
\hline & $\mathrm{BG}(k=-1)$ & $0.01565(0.09855)$ & $0.01774(0.10563)$ & $0.01565(0.09976)$ & $0.01721(0.10453)$ \\
\hline & $\mathrm{BG}(k=2)$ & $0.01495(0.09495)$ & $0.01613(0.10047)$ & $0.01549(0.09792)$ & $0.01722(0.10326)$ \\
\hline & $\mathrm{BG}(k=-2)$ & $0.01589(0.09983)$ & $0.01686(0.10303)$ & $0.01549(0.09711)$ & $0.01812(0.10809)$ \\
\hline \multirow{10}{*}{50} & ML & $0.01179(0.08736)$ & $0.01370(0.09561)$ & $0.01155(0.08619)$ & $0.01274(0.09169)$ \\
\hline & BS & $0.01180(0.08741)$ & $0.01371(0.09566)$ & $0.01156(0.08624)$ & $0.01276(0.09174)$ \\
\hline & $\mathrm{BL}(c=1)$ & $0.01160(0.08666)$ & $0.01354(0.09606)$ & $0.01164(0.08681)$ & $0.01299(0.09248)$ \\
\hline & $\mathrm{BL}(c=-1)$ & $0.01137(0.08563)$ & $0.01246(0.09134)$ & $0.01144(0.08683)$ & $0.01299(0.09263)$ \\
\hline & $\mathrm{BL}(c=2)$ & $0.01180(0.08794)$ & $0.01275(0.09056)$ & $0.01199(0.08865)$ & $0.01282(0.09201)$ \\
\hline & $\operatorname{BL}(c=-2)$ & $0.01192(0.08840)$ & $0.01353(0.09489)$ & $0.01197(0.08823)$ & 0.01239 (0.09009) \\
\hline & $\mathrm{BG}(k=1)$ & $0.01179(0.08768)$ & $0.01312(0.09299)$ & $0.01139(0.08609)$ & $0.01263(0.09120)$ \\
\hline & $\mathrm{BG}(k=-1)$ & $0.01799(0.08722)$ & $0.01259(0.09168)$ & $0.01109(0.08408)$ & $0.01274(0.09257)$ \\
\hline & $\mathrm{BG}(k=2)$ & $0.01143(0.08651)$ & $0.01320(0.09432)$ & $0.01136(0.08635)$ & $0.01273(0.09260)$ \\
\hline & $\mathrm{BG}(k=-2)$ & $0.01159(0.08686)$ & $0.01229(0.09049)$ & $0.01133(0.08472)$ & $0.01306(0.09325)$ \\
\hline \multirow{10}{*}{100} & ML & $0.00969(0.08234)$ & $0.01108(0.08929)$ & $0.00915(0.07937)$ & $0.01119(0.08962)$ \\
\hline & BS & $0.00969(0.08235)$ & $0.01108(0.08931)$ & $0.00715(0.07938)$ & $0.01119(0.08963)$ \\
\hline & $\mathrm{BL}(c=1)$ & $0.00953(0.08105)$ & $0.01109(0.08969)$ & $0.00932(0.08059)$ & $0.01136(0.09084)$ \\
\hline & $\mathrm{BL}(c=-1)$ & $0.00938(0.08148)$ & $0.01062(0.08779)$ & $0.00952(0.08159)$ & $0.01056(0.08705)$ \\
\hline & $\mathrm{BL}(c=2)$ & $0.00945(0.08189)$ & $0.01087(0.08877)$ & $0.00746(0.08212)$ & 0.01067 (0.08779) \\
\hline & $\mathrm{BL}(c=-2)$ & $0.00970(0.08226)$ & $0.01111(0.08950)$ & $0.00943(0.08102)$ & $0.01078(0.08804)$ \\
\hline & $\mathrm{BG}(k=1)$ & $0.00951(0.08202)$ & $0.01132(0.09110)$ & $0.00916(0.07991)$ & $0.01124(0.09032)$ \\
\hline & $\mathrm{BG}(k=-1)$ & $0.00958(0.08190)$ & $0.01085(0.08838)$ & $0.00933(0.08117)$ & $0.01071(0.08810)$ \\
\hline & BG $(k=2)$ & $0.00905(0.07915)$ & $0.01118(0.08959)$ & $0.00845(0.08126)$ & $0.01068(0.08739)$ \\
\hline & $\mathrm{BG}(k=-2)$ & $0.00893(0.07912)$ & $0.01071(0.08751)$ & $0.00946(0.08176)$ & $0.01124(0.09051)$ \\
\hline
\end{tabular}

ML: maximum likelihood, BG: general entropy loss function, BL: LINEX loss function, BS: squared error loss function.

\section{Results and Discussion}

From Tables 1 and 3, the most dominant estimator that had the smallest mean squared error is the Bayesian under linear exponential loss function(LINEX). This happened with generalised noninformative prior except at $n=50$ with $\alpha=1.5$ and $\beta=1.2$ that we observed that the noninformative gamma prior gave the smallest MSE. This was followed closely by the general entropy loss function (GELF) with the noninformative gamma prior. What is remarkable is that the smallest absolute bias values occurred mostly with the generalised noninformative prior. GELF was slightly ahead of LINEX but both were better than SELF and that of the MLE. 
Table 2: MSEs and Abs. Biases (in parenthesis) for the failure rate $F(t)$.

\begin{tabular}{|c|c|c|c|c|c|}
\hline \multirow{2}{*}{$n$} & & \multicolumn{2}{|c|}{$\alpha=0.5$} & \multicolumn{2}{|c|}{$\alpha=1.5$} \\
\hline & & $\beta=0.8$ & $\beta=1.2$ & $\beta=0.8$ & $\beta=1.2$ \\
\hline \multirow{10}{*}{25} & ML & $3.68099(0.54756)$ & $0.67105(0.70131)$ & $0.32433(0.22685)$ & $0.08476(0.24458)$ \\
\hline & BS & $3.68274(0.54755)$ & $0.67104(0.70129)$ & $0.32525(0.22687)$ & $0.08476(0.24581)$ \\
\hline & $\mathrm{BL}(c=1)$ & $2.90228(0.64591)$ & $0.73442(0.73753)$ & $0.22901(0.21768)$ & $0.08163(0.24202)$ \\
\hline & $\mathrm{BL}(c=-1)$ & $3.26232(0.65045)$ & $0.73879(0.72318)$ & $0.83318(0.22460)$ & $0.07709(0.23813)$ \\
\hline & $\mathrm{BL}(c=2)$ & $2.82598(0.65421)$ & $0.73698(0.73324)$ & $0.46876(0.21809)$ & $0.07626(0.23769)$ \\
\hline & $\mathrm{BL}(c=-2)$ & $2.05587(0.66813)$ & $0.71049(0.71956)$ & $0.28028(0.22308)$ & $0.07876(0.23947)$ \\
\hline & $\mathrm{BG}(k=1)$ & $3.45630(0.64855)$ & $0.70479(0.72267)$ & $0.51944(0.21948)$ & $0.07729(0.23826)$ \\
\hline & BG $(k=-1)$ & $3.38251(0.66240)$ & $0.68825(0.71194)$ & $0.43598(0.21791)$ & $0.07829(0.23991)$ \\
\hline & $\mathrm{BG}(k=2)$ & $2.74521(0.68257)$ & $0.68329(0.70471)$ & $0.53684(0.22410)$ & $0.07949(0.24022)$ \\
\hline & $\mathrm{BG}(k=-2)$ & $2.63739(0.67368)$ & $0.64373(0.69148)$ & $0.32230(0.21216)$ & $0.08217(0.24390)$ \\
\hline \multirow{10}{*}{50} & $\mathrm{ML}$ & $2.74229(0.54756)$ & $0.55963(0.66088)$ & $0.10479(0.18498)$ & $0.05991(0.21456)$ \\
\hline & BS & $2.74207(0.54755)$ & $0.55962(0.66087)$ & $0.10482(0.18497)$ & $0.05991(0.21246)$ \\
\hline & $\mathrm{BL}(c=1)$ & $2.06952(0.56294)$ & $0.55590(0.66412)$ & $0.09266(0.18624)$ & $0.06056(0.21627)$ \\
\hline & $\mathrm{BL}(c=-1)$ & $2.07558(0.55519)$ & $0.54833(0.65331)$ & $0.10693(0.18579)$ & $0.06066(0.21665)$ \\
\hline & $\mathrm{BL}(c=2)$ & $1.66649(0.56345)$ & $0.54094(0.64491)$ & $0.08616(0.18786)$ & $0.06103(0.21764)$ \\
\hline & $\mathrm{BL}(c=-2)$ & $1.48847(0.56854)$ & $0.57137(0.66531)$ & $0.11013(0.18562)$ & $0.05871(0.21287)$ \\
\hline & $\mathrm{BG}(k=1)$ & $2.35703(0.57154)$ & $0.55850(0.65845)$ & $0.09676(0.18616)$ & $0.05904(0.21293)$ \\
\hline & $\mathrm{BG}(k=-1)$ & $1.06927(0.56011)$ & $0.54374(0.64790)$ & $0.16384(0.18465)$ & $0.06124(0.21868)$ \\
\hline & $\mathrm{BG}(k=2)$ & $1.05864(0.55842)$ & $0.56163(0.66459)$ & $0.08869(0.18615)$ & $0.06057(0.21822)$ \\
\hline & $\mathrm{BG}(k=-2)$ & $0.85649(0.55619)$ & $0.52414(0.63780)$ & $0.08375(0.18829)$ & $0.06088(0.21713)$ \\
\hline \multirow{10}{*}{100} & $\mathrm{ML}$ & $0.67729(0.53509)$ & $0.50136(0.64635)$ & $0.05015(0.17142)$ & $0.05539(0.21498)$ \\
\hline & BS & $0.67733(0.53509)$ & $0.50136(0.64635)$ & $0.05015(0.17142)$ & $0.05539(0.21498)$ \\
\hline & $\mathrm{BL}(c=1)$ & $0.45556(0.52062)$ & $0.49361(0.64479)$ & $0.06047(0.17411)$ & $0.05607(0.21656)$ \\
\hline & $\mathrm{BL}(c=-1)$ & $0.42816(0.52640)$ & $0.47733(0.63167)$ & $0.09317(0.17839)$ & $0.05342(0.21074)$ \\
\hline & $\mathrm{BL}(c=2)$ & $0.48344(0.52865)$ & $0.49163(0.64201)$ & $0.04895(0.17618)$ & $0.05405(0.21265)$ \\
\hline & $\mathrm{BL}(c=-2)$ & $0.47254(0.52719)$ & $0.49932(0.64575)$ & $0.05123(0.17538)$ & $0.05406(0.21182)$ \\
\hline & $\mathrm{BG}(k=1)$ & $0.44526(0.52608)$ & $0.50362(0.65328)$ & $0.05159(0.17252)$ & $0.05432(0.21427)$ \\
\hline & $\mathrm{BG}(k=-1)$ & $0.51433(0.53095)$ & $0.48884(0.63931)$ & $0.06519(0.17545)$ & $0.05395(0.21272)$ \\
\hline & $\mathrm{BG}(k=2)$ & $0.44763(0.51844)$ & $0.50123(0.64629)$ & $0.07165(0.17638)$ & $0.05428(0.21193)$ \\
\hline & $\mathrm{BG}(k=-2)$ & $0.46723(0.51550)$ & $0.48396(0.63387)$ & $0.05236(0.17513)$ & $0.05526(0.21548)$ \\
\hline
\end{tabular}

ML: maximum likelihood, BG: general entropy loss function, BL: LINEX loss function, BS: squared error loss function.

The implication from above is that the generalised noninformative prior worked remarkable well under both LINEX and GELF with respect to the mean squared error and absolute bias. What need to be stated is that the generalised prior ensures that since there is very little knowledge on the function being estimated as a result of which a noninformative prior is assumed and obtained from the available data via MLE, then one should ensure that at the end it plays little role so that the Bayesian inference is based on the available data.

Considering Tables 2 and 4, we noticed again that the generalised prior performed astonishingly well against the noninformative prior under both the GELF and LINEX. Both priors performed equally well under general entropy and linear exponential loss functions. With the absolute bias values, the generalised noninformative prior incredibly performed 
Table 3: MSEs and Abs. Biases (in parenthesis) for the survival function $S(t)$ using GNP.

\begin{tabular}{|c|c|c|c|c|c|}
\hline \multirow{3}{*}{\multicolumn{2}{|c|}{$n$}} & \multicolumn{2}{|c|}{$\alpha=0.5$} & \multicolumn{2}{|c|}{$\alpha=1.5$} \\
\hline & & $\beta=0.8$ & $\beta=1.2$ & $\beta=0.8$ & \\
\hline & & \multicolumn{2}{|c|}{$a=5,[3]$} & \multicolumn{2}{|c|}{$a=5,[3]$} \\
\hline \multirow{18}{*}{25} & \multirow{2}{*}{ BS } & $0.01671(0.10237)$ & $0.01731(0.10550)$ & $0.01553(0.09804)$ & $0.01740(0.10389)$ \\
\hline & & {$[0.01528(0.09853)]$} & [0.01733 (0.10441)] & [0.01632 (0.10123)] & [0.01861 (0.10795)] \\
\hline & \multirow{2}{*}{$\mathrm{BL}(c=1)$} & $0.01736(0.01025)$ & $0.01608(0.10052)$ & $0.01531(0.09805)$ & $0.01694(0.10227)$ \\
\hline & & [0.01559 (0.09876)] & {$[0.01726(0.10385)]$} & [0.01585 (0.09796)] & [0.01755 (0.10476)] \\
\hline & \multirow{2}{*}{$\mathrm{BL}(c=-1)$} & $0.01756(0.10315)$ & $0.01655(0.10308)$ & $0.01614(0.10075)$ & $0.01748(0.10470)$ \\
\hline & & [0.01544 (0.09803)] & {$[0.01806(0.10735)]$} & [0.01680 (0.10254)] & {$[0.01727(0.10453)]$} \\
\hline & \multirow{2}{*}{$\mathrm{BL}(c=2)$} & $0.01797(0.10635)$ & $0.01649(0.10116)$ & $0.01467(0.09588)$ & $0.01718(0.10317)$ \\
\hline & & [0.01716 (0.10194)] & {$[0.01806(0.10617)]$} & [0.01506 (0.09667)] & [0.01793 (0.10640)] \\
\hline & \multirow{2}{*}{$\mathrm{BL}(c=-2)$} & $0.01756(0.10499)$ & $0.01637(0.10090)$ & $0.01571(0.09796)$ & $0.01748(0.10551)$ \\
\hline & & [0.01554 (0.09817)] & [0.01732 (0.10397)] & {$[0.01602(0.10003)]$} & [0.01857 (0.10914)] \\
\hline & \multirow{2}{*}{$\mathrm{BG}(k=1)$} & $0.01645(0.10157)$ & $0.01659(0.10271)$ & $0.01587(0.09919)$ & $0.01719(0.10355)$ \\
\hline & & {$[0.01519(0.09767)]$} & {$[0.01741(0.10468)]$} & [0.01649 (0.10107)] & [0.01782 (0.10583)] \\
\hline & \multirow{2}{*}{$\mathrm{BG}(k=-1)$} & $0.01660(0.10067)$ & $0.01734(0.10515)$ & $0.01621(0.09978)$ & $0.01808(0.10817)$ \\
\hline & & [0.01554 (0.09757)] & [0.01609 (0.10096)] & {$[0.01539(0.09644)]$} & [0.01793 (0.10731)] \\
\hline & \multirow{2}{*}{$\mathrm{BG}(k=2)$} & $0.01794(0.10643)$ & $0.01773(0.10477)$ & $0.01653(0.10009)$ & $0.01688(0.10393)$ \\
\hline & & [0.01551 (0.09821)] & {$[0.01690(0.10249)]$} & {$[0.01518(0.09757)]$} & [0.01667 (0.10306)] \\
\hline & \multirow{2}{*}{$\mathrm{BG}(k=-2)$} & $0.01623(0.10158)$ & $0.01730(0.10519)$ & $0.01562(0.09759)$ & $0.01748(0.10465)$ \\
\hline & & {$[0.01654(0.10175)]$} & {$[0.01773(0.10552)]$} & {$[0.01636(0.10043)]$} & {$[0.01822(0.10797)]$} \\
\hline \multirow{18}{*}{50} & \multirow{2}{*}{ BS } & $0.01178(0.08765)$ & $0.01299(0.09209)$ & $0.01174(0.08754)$ & $0.01312(0.09343)$ \\
\hline & & [0.01163 (0.08629)] & [0.01255 (0.09154)] & [0.01163 (0.08679)] & [0.01272 (0.09223)] \\
\hline & \multirow{2}{*}{$\mathrm{BL}(c=1)$} & $0.01092(0.08456)$ & $0.01259(0.09203)$ & $0.01134(0.08587)$ & $0.01276(0.09198)$ \\
\hline & & {$[0.01128(0.08459)]$} & {$[0.01284(0.09326)]$} & {$[0.01181(0.08617)]$} & {$[0.01289(0.09290)]$} \\
\hline & \multirow{2}{*}{$\mathrm{BL}(c=-1)$} & $0.01201(0.08874)$ & $0.01266(0.09119)$ & $0.01149(0.08649)$ & $0.01270(0.09149)$ \\
\hline & & [0.01118 (0.08561)] & [0.01274 (0.09196)] & [0.01126 (0.08554)] & [0.01251 (0.09036)] \\
\hline & \multirow{2}{*}{$\mathrm{BL}(c=2)$} & $0.01126(0.08480)$ & $0.01288(0.09267)$ & $0.01164(0.08756)$ & $0.01307(0.09326)$ \\
\hline & & {$[0.01149(0.08640)]$} & {$[0.01261(0.09249)]$} & {$[0.01106(0.08521)]$} & [0.01335 (0.09481)] \\
\hline & \multirow{2}{*}{$\mathrm{BL}(c=-2)$} & $0.01119(0.08567)$ & $0.01304(0.09343)$ & $0.01135(0.08484)$ & $0.01299(0.09379)$ \\
\hline & & [0.01158 (0.08676)] & [0.01234 (0.09038)] & {$[0.01209(0.08853)]$} & {$[0.01310(0.09310)]$} \\
\hline & \multirow{2}{*}{$\mathrm{BG}(k=1)$} & $0.01109(0.08496)$ & $0.01322(0.09375)$ & $0.01137(0.08565)$ & $0.01309(0.09474)$ \\
\hline & & {$[0.01111(0.08467)]$} & [0.01287 (0.09549)] & [0.01176 (0.08690)] & [0.01279 (0.09197)] \\
\hline & \multirow{2}{*}{$\mathrm{BG}(k=-1)$} & $0.01187(0.08821)$ & $0.01365(0.09533)$ & $0.01191(0.08832)$ & $0.01263(0.09130)$ \\
\hline & & [0.01147 (0.08608)] & {$[0.01289(0.09229)]$} & {$[0.01126(0.08621)]$} & [0.01298 (0.09259)] \\
\hline & & $0.01135(0.08535)$ & $0.01307(0.09349)$ & $0.01126(0.08878)$ & $0.01361(0.09489)$ \\
\hline & & {$[0.01197(0.08815)]$} & {$[0.01257(0.09113)]$} & [0.01145 (0.08588)] & [0.01251 (0.09138)] \\
\hline & & 0.01199 (0.08802) & $0.01325(0.09363)$ & $0.01232(0.08958)$ & $0.01259(0.09138)$ \\
\hline & & {$[0.01166(0.08674)]$} & {$[0.01299(0.09296)]$} & {$[0.01138(0.08564)]$} & [0.01302(0.09359)] \\
\hline & BS & $0.00967(0.08273)$ & $0.01069(0.08780)$ & $0.00935(0.08153)$ & $0.01107(0.08928)$ \\
\hline & DU & {$[0.00936(0.08143)]$} & {$[0.01087(0.08831)]$} & {$[0.00929(0.08039)]$} & {$[0.01084(0.08928)]$} \\
\hline & & $0.00984(0.08327)$ & $0.01084(0.08875)$ & $0.00867(0.07797)$ & $0.01061(0.08743)$ \\
\hline & & {$[0.00961(0.08267)]$} & [0.01090 (0.08894)] & {$[0.00969(0.08240)]$} & [0.01093 (0.08894)] \\
\hline & & $0.00951(0.08128)$ & $0.01051(0.08688)$ & $0.00975(0.08276)$ & 0.01119 (0.08929) \\
\hline 100 & & [0.00925 (0.08078)] & {$[0.01043(0.08648)]$} & {$[0.00963(0.08201)]$} & {$[0.01110(0.08923)]$} \\
\hline & & $0.00937(0.08062)$ & $0.01064(0.08733)$ & $0.00929(0.08096)$ & $0.01105(0.08896)$ \\
\hline & & [0.00973 (0.08233)] & [0.01101 (0.08887)] & [0.00969 (0.08302)] & [0.01118 (0.09015)] \\
\hline
\end{tabular}


Table 3: Continued.

\begin{tabular}{|c|c|c|c|c|}
\hline \multirow{3}{*}{$n$} & \multicolumn{2}{|c|}{$\alpha=0.5$} & \multicolumn{2}{|c|}{$\alpha=1.5$} \\
\hline & $\beta=0.8$ & $\beta=1.2$ & $\beta=0.8$ & $\beta=1.2$ \\
\hline & \multicolumn{2}{|c|}{$a=5,[3]$} & \multicolumn{2}{|c|}{$a=5,[3]$} \\
\hline \multirow{2}{*}{$\mathrm{BL}(c=-2)$} & $0.00952(0.08184)$ & $0.01098(0.08906)$ & $0.00966(0.08244)$ & $0.01087(0.08856)$ \\
\hline & [0.00994 (0.08372)] & {$[0.01102(0.08887)]$} & [0.00938 (0.08141)] & [0.01094 (0.08877)] \\
\hline \multirow{2}{*}{$\mathrm{BG}(k=1)$} & $0.00895(0.07874)$ & $0.01091(0.08838)$ & $0.00946(0.08088)$ & $0.01059(0.08729)$ \\
\hline & [0.00926 (0.08019)] & [0.01093 (0.08855)] & [0.00955 (0.08214)] & [0.01108 (0.08918)] \\
\hline \multirow{2}{*}{$\mathrm{BG}(k=-1)$} & $0.00914(0.08047)$ & $0.01093(0.08833)$ & $0.00956(0.08156)$ & $0.01078(0.08802)$ \\
\hline & [0.00897 (0.07956)] & [0.01091 (0.08844)] & [0.00985 (0.08260)] & [0.01001 (0.08491)] \\
\hline \multirow{2}{*}{$\mathrm{BG}(k=2)$} & $0.00929(0.08071)$ & $0.01106(0.08889)$ & $0.00921(0.07979)$ & $0.01142(0.09069)$ \\
\hline & [0.00963 (0.08220)] & {$[0.01111(0.08947)]$} & [0.00975 (0.08284)] & {$[0.01092(0.08865)]$} \\
\hline \multirow{2}{*}{$\mathrm{BG}(k=-2)$} & $0.00936(0.08122)$ & $0.01128(0.09042)$ & $0.00990(0.08379)$ & $0.01111(0.08941)$ \\
\hline & {$[0.00961(0.08213)]$} & {$[0.01072(0.08811)]$} & {$[0.00947(0.08152)]$} & {$[0.01132(0.09072)]$} \\
\hline
\end{tabular}

GNP: generalised nonInformative prior, BG: general entropy loss function, BL: LINEX loss function, BS: squared error loss function.

better under LINEX loss function but was almost equal with the noninformative prior under the general entropy loss function.

To obtain the MSE values for each estimated value, the MSE is calculated for each of the one thousand estimated values of the survival function and the failure rate that is, from 1 to 1000 . At the end, we obtain the average of the MSE, values. Our aim is to find out how close the estimated values of the estimators are to the true value. The absolute bias values are obtained in like manner and of course from the same simulated values as that of the MSE.

\section{Conclusion}

In this study, we consider the point estimation of the Weibull distribution based on right censoring through simulation. MLE and Bayes estimators are applied to estimate the survival function and the failure rate of this lifetime distribution. The Bayes estimators are obtained using linear exponential, general entropy, and squared error loss functions. We also employed the Bayesian noninformative prior approach in estimating survival function and the failure rate. In order to reduce the complicated integrals that are in the posterior distribution which cannot explicitly be obtained in close form, we employed the Lindley approximation procedure to calculate the Bayes estimators.

Another point worth noting is that we assumed an informative prior for the scale parameter and the shape parameter which led us into obtaining an improper prior. We also made a proposition for a generalised noninformative prior.

From the results and discussions above, it is evident that the proposed generalised noninformative prior performed quite well than the noninformative gamma prior. In all the cases, the Bayesian estimator using the generalised noninformative prior under the linear exponential loss function overall performed better than the other estimators and under the other different loss functions with respect to the mean squared error. It has also been observed that the smallest absolute bias values occurred predominantly with the generalised 
Table 4: MSEs and Abs. Biases (in parenthesis) for the failure rate $F(t)$ using GNP.

\begin{tabular}{|c|c|c|c|c|c|}
\hline \multirow{3}{*}{\multicolumn{2}{|c|}{$n$}} & \multicolumn{2}{|c|}{$\alpha=0.5$} & \multicolumn{2}{|c|}{$\alpha=1.5$} \\
\hline & & $\beta=0.8$ & $\beta=1.2$ & $\beta=0.8$ & $\beta=1.2$ \\
\hline & & \multicolumn{2}{|c|}{$a=5,[3]$} & \multicolumn{2}{|c|}{$a=5,[3]$} \\
\hline \multirow{18}{*}{25} & \multirow{2}{*}{ BS } & $1.60882(0.60581)$ & $0.75601(0.74247)$ & $0.26918(0.22161)$ & $0.08242(0.24192)$ \\
\hline & & [3.50808 (0.66123)] & [0.70983 (0.72011)] & {$[0.21814(0.22270)]$} & {$[0.08227(0.24568)]$} \\
\hline & \multirow{2}{*}{$\mathrm{BL}(c=1)$} & $3.10223(0.66497)$ & $0.66435(0.70130)$ & $0.24086(0.21740)$ & $0.07698(0.23560)$ \\
\hline & & [0.97145 (0.64334)] & {$[0.73049(0.73445)]$} & [0.46015 (0.22941)] & [0.08183 (0.24475)] \\
\hline & \multirow{2}{*}{$\mathrm{BL}(c=-1)$} & $5.07183(0.70026)$ & $0.72658(0.71728)$ & $0.17702(0.22059)$ & $0.07997(0.24249)$ \\
\hline & & [1.89290 (0.65885)] & {$[0.72855(0.73625)]$} & {$[0.28853(0.22911)]$} & {$[0.07864(0.23852)]$} \\
\hline & \multirow{2}{*}{$\mathrm{BL}(c=2)$} & $2.57595(0.67277)$ & $0.70089(0.71957)$ & $0.19602(0.21443)$ & $0.08062(0.24190)$ \\
\hline & & {$[1.42447(0.65551)]$} & {$[0.73245(0.73581)]$} & {$[0.26676(0.22149)]$} & {$[0.08261(0.24604)]$} \\
\hline & \multirow{2}{*}{$\mathrm{BL}(c=-2)$} & $2.47065(0.68677)$ & $0.68712(0.70818)$ & $0.29206(0.22263)$ & $0.07838(0.24087)$ \\
\hline & & [2.90974 (0.65568)] & {$[0.69822(0.71461)]$} & {$[0.31298(0.23181)]$} & {$[0.08179(0.24609)]$} \\
\hline & \multirow{2}{*}{$\mathrm{BG}(k=1)$} & $5.09142(0.63923)$ & $0.68482(0.71454)$ & $0.32629(0.21537)$ & $0.07963(0.24117)$ \\
\hline & & [1.59876 (0.64264)] & {$[0.71224(0.72443)]$} & [0.39187 (0.22194)] & [0.08104 (0.24269)] \\
\hline & \multirow{2}{*}{$\mathrm{BG}(k=-1)$} & $1.11653(0.60663)$ & $0.73329(0.72535)$ & $0.28561(0.21867)$ & $0.07938(0.24085)$ \\
\hline & & {$[2.05799(0.65800)]$} & [0.67003 (0.70302)] & {$[0.28235(0.28235)]$} & {$[0.07783(0.24017)]$} \\
\hline & \multirow{2}{*}{$\mathrm{BG}(k=2)$} & $3.26989(0.63433)$ & $0.70409(0.72102)$ & $0.14943(0.21824)$ & $0.08079(0.24219)$ \\
\hline & & [3.59616 (0.65300)] & {$[0.70879(0.71412)]$} & [0.20827 (0.22005)] & [0.08084 (0.24227)] \\
\hline & \multirow{2}{*}{$\mathrm{BG}(k=-2)$} & $1.56123(0.63166)$ & $0.68200(0.71084)$ & $0.22126(0.21289)$ & $0.07983(0.24229)$ \\
\hline & & [1.82844 (0.64736)] & {$[0.71757(0.72167)]$} & [0.16709 (0.21693)] & [0.07978 (0.24356)] \\
\hline \multirow{18}{*}{50} & \multirow{2}{*}{ BS } & $0.99284(0.55903)$ & $0.53895(0.64125)$ & $0.21602(0.18856)$ & $0.06088(0.21759)$ \\
\hline & & {$[1.20392(0.55531)]$} & {$[0.53767(0.64578)]$} & {$[0.23509(0.18781)]$} & {$[0.05879(0.21411)]$} \\
\hline & \multirow{2}{*}{$\mathrm{BL}(c=1)$} & $0.85858(0.55004)$ & $0.54626(0.65313)$ & $0.21497(0.18676)$ & $0.06021(0.21590)$ \\
\hline & & [0.84299 (0.54806)] & [0.54644 (0.65612)] & [0.18170 (0.18565)] & [0.06051 (0.21811)] \\
\hline & \multirow{2}{*}{$\mathrm{BL}(c=-1)$} & $0.90621(0.56868)$ & $0.55652(0.65539)$ & $0.17717(0.18777)$ & $0.06052(0.21565)$ \\
\hline & & {$[0.70963(0.54405)]$} & {$[0.53659(0.64556)]$} & {$[0.19359(0.18475)]$} & {$[0.06063(0.21566)]$} \\
\hline & \multirow{2}{*}{$\mathrm{BL}(c=2)$} & $0.84116(0.54422)$ & $0.55124(0.65304)$ & $0.19584(0.18896)$ & $0.06132(0.21808)$ \\
\hline & & [1.05227 (0.55656)] & {$[0.55016(0.65747)]$} & [0.19113 (0.18416)] & {$[0.06118(0.21996)]$} \\
\hline & \multirow{2}{*}{$\mathrm{BL}(c=-2)$} & $0.83699(0.55410)$ & $0.54021(0.64881)$ & $0.19281(0.18479)$ & $0.06111(0.21948)$ \\
\hline & & [0.87199 (0.55693)] & [0.52738 (0.63962)] & [0.18451 (0.18884)] & {$[0.06072(0.21715)]$} \\
\hline & \multirow{2}{*}{$\mathrm{BG}(k=1)$} & $1.08699(0.55039)$ & $0.55381(0.65826)$ & $0.17302(0.18223)$ & $0.06119(0.21882)$ \\
\hline & & [0.95041 (0.55106)] & [0.54985 (0.65467)] & [0.24233 (0.18548)] & [0.06097 (0.21819)] \\
\hline & \multirow{2}{*}{$\mathrm{BG}(k=-1)$} & $1.12507(0.55889)$ & $0.55956(0.66129)$ & $0.25057(0.19005)$ & $0.06023(0.21461)$ \\
\hline & & [1.01425 (0.55157)] & {$[0.55221(0.65488)]$} & {$[0.11878(0.18655)]$} & {$[0.06018(0.21560)]$} \\
\hline & & 1.17354 (0.55312) & $0.57334(0.67120)$ & $0.17699(0.18661)$ & $0.06244(0.22011)$ \\
\hline & & [1.00135 (0.56841)] & {$[0.54657(0.64917)]$} & {$[0.10609(0.18484)]$} & {$[0.05985(0.21521)]$} \\
\hline & $\mathrm{BG}(k=-2)$ & $1.18804(0.56841)$ & $0.56341(0.66432)$ & $0.13249(0.18605)$ & $0.06024(0.21510)$ \\
\hline & & {$[1.15358(0.55573)]$} & {$[0.54699(0.65363)]$} & {$[0.14781(0.18359)]$} & {$[0.06057(0.21754)]$} \\
\hline & $B S$ & $0.66670(0.53230)$ & $0.48857(0.63609)$ & $0.05482(0.17604)$ & $0.05524(0.21483)$ \\
\hline & & {$[0.44568(0.52663)]$} & [0.49065 (0.64062)] & [0.06864 (0.17410)] & {$[0.05323(0.21001)]$} \\
\hline & & $0.47245(0.53712)$ & $0.48375(0.63740)$ & $0.04677(0.16927)$ & $0.05397(0.21152)$ \\
\hline & & {$[0.48126(0.52985)]$} & {$[0.49223(0.64333)]$} & [0.06145 (0.17697)] & [0.05467 (0.21400)] \\
\hline & $\mathrm{BL}(c=-1)$ & $0.50560(0.52798)$ & $0.48800(0.63826)$ & $0.06408(0.17864)$ & $0.05512(0.21358)$ \\
\hline 100 & & {$[0.48709(0.52132)]$} & {$[0.47258(0.62645)]$} & [0.06531 (0.17577)] & [0.05552 (0.21507)] \\
\hline & & $0.44645(0.52047)$ & $0.48540(0.63612)$ & $0.06605(0.17422)$ & $0.05524(0.21454)$ \\
\hline & & {$[0.80817(0.52984)]$} & {$[0.48774(0.63795)]$} & [0.06651 (0.17786)] & {$[0.05648(0.21780)]$} \\
\hline
\end{tabular}


Table 4: Continued.

\begin{tabular}{cccccc}
\hline \multirow{2}{n}{$n$} & \multicolumn{3}{c}{$\alpha=0.5$} & \multicolumn{2}{c}{$\alpha=1.5$} \\
& \multicolumn{2}{c}{$a=5,[3]$} & $\beta=1.2$ & $\beta=0.8$ & $\beta=1.2$ \\
& & $0.50473(0.52856)$ & $0.49342(0.64368)$ & $0.05039(0.17666)$ & $0.05434(0.21327)$ \\
BL $(c=-2)$ & {$[0.52768(0.53450)]$} & {$[0.49421(0.64037)]$} & {$[0.07692(0.17494)]$} & {$[0.05561(0.21493)]$} \\
& $0.44440(0.51305)$ & $0.49572(0.64148)$ & $0.07150(0.17498)$ & $0.05361(0.21112)$ \\
BG $(k=1)$ & {$[0.59699(0.52223)]$} & {$[0.49308(0.64049)]$} & {$[0.05309(0.17608)]$} & {$[0.05444(0.21287)]$} \\
& $0.49145(0.52292)$ & $0.49925(0.64372)$ & $0.05013(0.17478)$ & $0.05340(0.21109)$ \\
BG $(k=-1)$ & {$[0.46108(0.52107)]$} & {$[0.49309(0.63951)]$} & {$[0.04829(0.17618)]$} & {$[0.05185(0.20729)]$} \\
& $0.50315(0.52624)$ & $0.50124(0.64500)$ & $0.05319(0.17324)$ & $0.05761(0.22003)$ \\
BG $(k=2)$ & {$[0.45561(0.52818)]$} & {$[0.49235(0.64167)]$} & {$[0.05073(0.17732)]$} & {$[0.05492(0.21381)]$} \\
& $0.55240(0.52938)$ & $0.51051(0.65583)$ & $0.04795(0.17732)$ & $0.05482(0.21406)$ \\
BG $(k=-2)$ & {$[0.42269(0.52581)]$} & {$[0.48926(0.63954)]$} & {$[0.06453(0.17769)]$} & {$[0.05630(0.21743)]$} \\
\hline
\end{tabular}

GNP: generalised noninformative prior, BG: general entropy loss function, BL: LINEX loss function, BS: squared error loss function.

noninformative prior under general entropy loss function for both the survival function and the failure rate.

\section{Acknowledgments}

The authors would like to thank the anonymous referees for their suggestions and comments. The first author would like to personally thank the Editor, Professor Carlo Cattani, for his encouragement and suggestions.

\section{References}

[1] J. F. Lawless, Statistical Models and Methods for Lifetime Data, John Wiley \& Sons, New York, NY, USA, 1982.

[2] H. Rinne, The Weibull Distribution, CRC Press, Taylor and Francis Group LLC, New York, NY, USA, 2009.

[3] S. K. Sinha, "Bayes estimation of the reliability function and hazard rate of a weibull failure time distribution," Tranbajos De Estadistica, vol. 1, pp. 47-56, 1986.

[4] A. A. Abdel-Wahid and A. Winterbottom, "Approximate Bayesian estimates for the Weibull reliability function and hazard rate from censored data," Journal of Statistical Planning and Inference, vol. 16, no. 3, pp. 277-283, 1987.

[5] M. A. Al Omari and N. A. Ibrahim, "Bayesian survival estimation estimation for weibull distribution with censored data," Journal of Applied Sciences, vol. 11, pp. 393-396, 2011.

[6] C. B. Guure, N. A. Ibrahim, and A. M. Al Omari, "Bayesian estimation of two-parameter weibull distribution using extension of Jeffreys' prior information with three loss functions," Mathematical Problems in Engineering, vol. 2012, Article ID 589640, 13 pages, 2012.

[7] H. Syuan-Rong and W. Shuo-Jye, "Bayesian estimation and prediction for weibull model with progressive censoring," Journal of Statistical Computation and Simulation, pp. 1-14, 2011.

[8] C. Cattani and A. Ciancio, "Separable transition density in the hybrid model for tumor-immune system competition," Computational and Mathematical Methods in Medicine, vol. 2012, Article ID 610124, 6 pages, 2012.

[9] X. Wang, M. Xia, C. Huiwen, Y. Gao, and C. Cattani, “Hidden-markov-models-based dynamic hand gesture recognition," Mathematical Problems in Engineering, vol. 2012, Article ID 986134, 11 pages, 2012.

[10] C. Cattani, "On the existence of wavelet symmetries in archaea DNA," Computational and Mathematical Methods in Medicine, vol. 2012, Article ID 673934, 21 pages, 2012.

[11] C. Cattani, "Fractional calculus and shannon wavelet," Mathematical Problems in Engineering, vol. 2012, Article ID 502812, 26 pages, 2012.

[12] R. D. Gupta and D. Kundu, "Generalized exponential distribution: different method of estimations," Journal of Statistical Computation and Simulation, vol. 69, no. 4, pp. 315-337, 2001. 
[13] A. Zellner, "Bayesian estimation and prediction using asymmetric loss functions," Journal of the American Statistical Association, vol. 81, no. 394, pp. 446-451, 1986.

[14] F. M. Al-Aboud, "Bayesian estimations for the extreme value distribution using progressive censored data and asymmetric loss," International Mathematical Forum, vol. 4, no. 33-36, pp. 1603-1622, 2009.

[15] F. M. Al-Athari, "Parameter estimation for the double-pareto distribution," Journal of Mathematics and Statistics, vol. 7, no. 4, pp. 289-294, 2011.

[16] C. Cattani, G. Pierro, and G. Altieri, "Entropy and multifractality for the myeloma multiple TET 2 gene," Mathematical Problems in Engineering, vol. 2012, Article ID 193761, 14 pages, 2012.

[17] B. N. Pandey, N. Dwividi, and B. Pulastya, "Comparison between Bayesian and maximum likelihood estimation of the scale parameter in weibull distribution with known shape under linex loss function," Journal of Scientific Research, vol. 55, pp. 163-172, 2011.

[18] D. Kundu and R. D. Gupta, "Generalized exponential distribution: bayesian estimations," Computational Statistics \& Data Analysis, vol. 52, no. 4, pp. 1873-1883, 2008.

[19] P. Vasile, P. Eugeine, and C. Alina, "Bayes estimators of modified-Weibull distribution parameters using Lindley's approximation," WSEAS Transactions on Mathematics, vol. 9, no. 7, pp. 539-549, 2010.

[20] A. P. Basu and N. Ebrahimi, "Bayesian approach to life testing and reliability estimation using asymmetric loss function," Journal of Statistical Planning and Inference, vol. 29, no. 1-2, pp. 21-31, 1991.

[21] J. O. Berger and D. C. Sun, "Bayesian analysis for the poly-Weibull distribution," Journal of the American Statistical Association, vol. 88, no. 424, pp. 1412-1418, 1993.

[22] A. Banerjee and D. Kundu, "Inference based on type-II hybrid censored data from a weibull distribution," IEEE Transactions on Reliability, vol. 57, no. 2, pp. 369-378, 2008.

[23] J. O. Berger, "The case for objective Bayesian analysis," Bayesian Analysis, vol. 1, no. 3, pp. 385-402, 2006.

[24] A. A. Soliman, A. H. Abd Ellah, and S. B. Ryan, "Comparison of estimates using record statistics from weibull model: bayesian and non-Bayesian approaches," Computational Statistics \& Data Analysis, vol. 51, pp. 2065-2077, 2006.

[25] D. V. Lindley, “Approximate Bayesian methods," Trabajos Estadist, vol. 31, pp. 223-237, 1980.

[26] R. Calabria and G. Pulcini, "Point estimation under asymmetric loss functions for left-truncated exponential samples," Communications in Statistics. Theory and Methods, vol. 25, no. 3, pp. 585-600, 1996. 


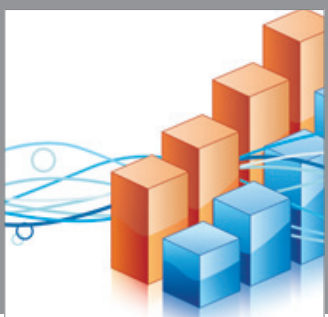

Advances in

Operations Research

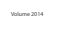

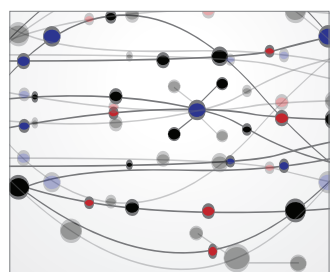

\section{The Scientific} World Journal
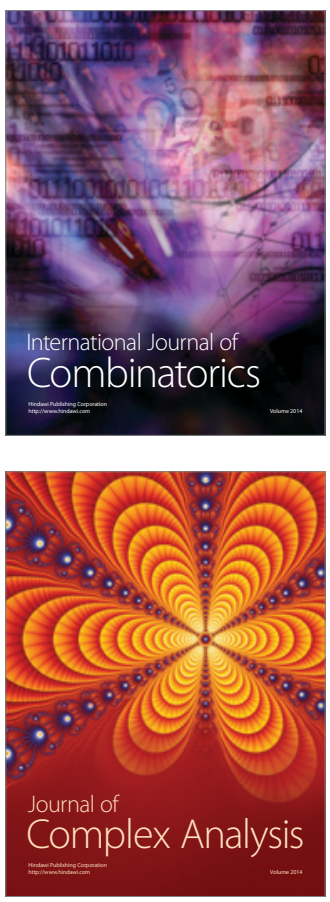

International Journal of

Mathematics and

Mathematical

Sciences
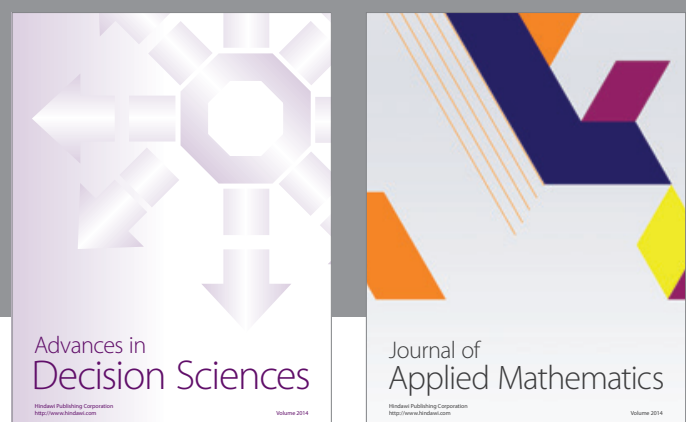

Journal of

Applied Mathematics
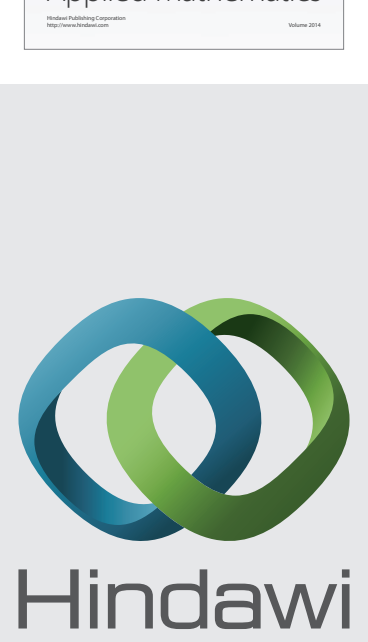

Submit your manuscripts at http://www.hindawi.com
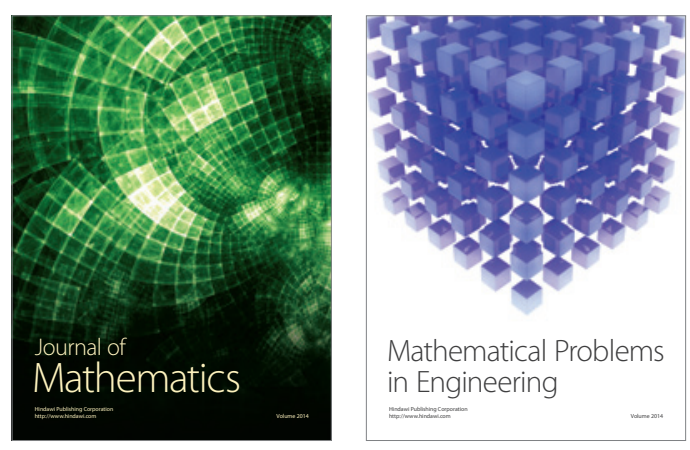

Mathematical Problems in Engineering
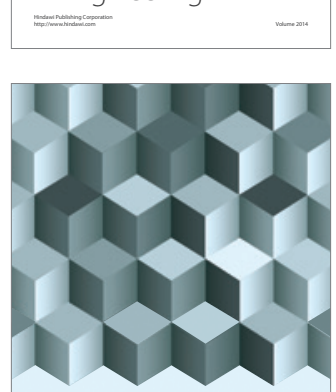

Journal of

Function Spaces
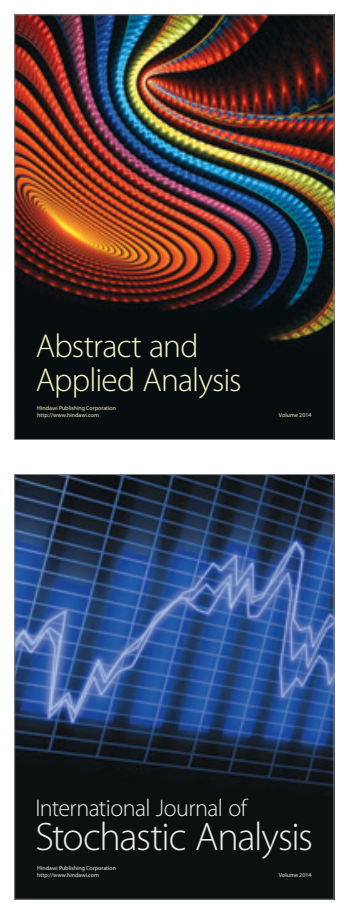

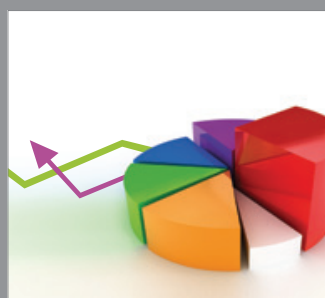

ournal of

Probability and Statistics

Promensencen
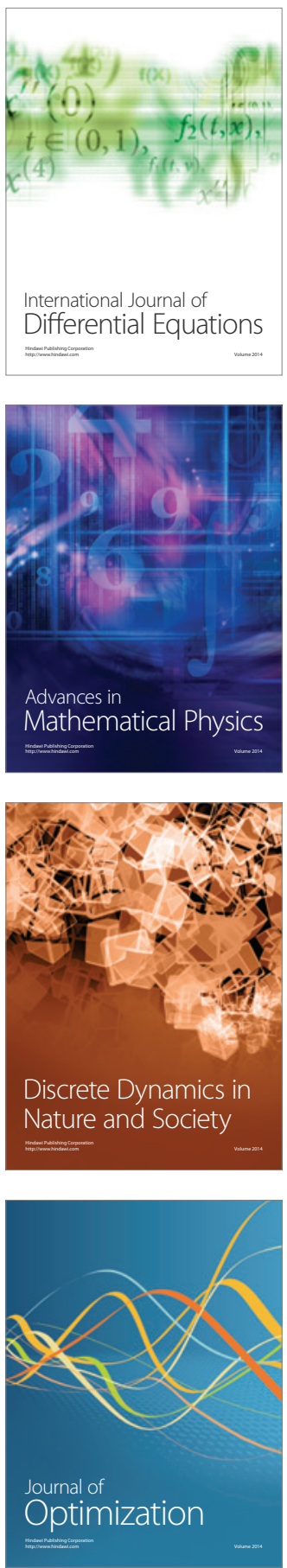